\title{
Axonal loss linked to MS disability
}

Chronic, irreversible motor disability in patients with multiple sclerosis (MS) correlates with axonal pathology rather than demyelination, according to new research. During the early stages of MS, patients mainly experience reversible disability, but in progressive MS the motor impairments become increasingly severe owing to cumulative tissue damage. Researcher Nikos Evangelou, of the University of Nottingham, UK, explains "in the causation of disability, what was missing was the direct evidence linking clinical disability measured in vivo with corresponding postmortem measures of axonal pathology."

Evangelou and his colleagues examined postmortem spinal cord tissue sections from 45 individuals with MS, while using medical records to gain a clear impression of the degree of motor disability that each patient had accumulated before death. Expanded Disability Status Scale scores were obtained, either directly or indirectly, from these records. The research team found that patients with progressive MS who had accumulated higher levels of motor disability before death had fewer surviving corticospinal tract axons. By contrast, no correlation was observed between the extent of demyelination and the degree of disability.

This new evidence builds on previous research indicating a primary role for axonal pathology in motor deterioration in animals. The outcome, says Evangelou, "stresses the importance of pathological studies in helping us understand a whole gamut of neuropsychiatric diseases." Commenting on the favorable sample size and information available to the team from UK and Netherlands-based Brain Banks, Evangelou points out "the need for tissue donations and support of brain banks is now more important than ever."

\section{Carolyn McSharry}

Original article Tallantyre, E. C. et al. Clinico-pathological evidence that axonal loss underlies disability in progressive multiple sclerosis. Mult. Scler. 16, 406-411 (2010)
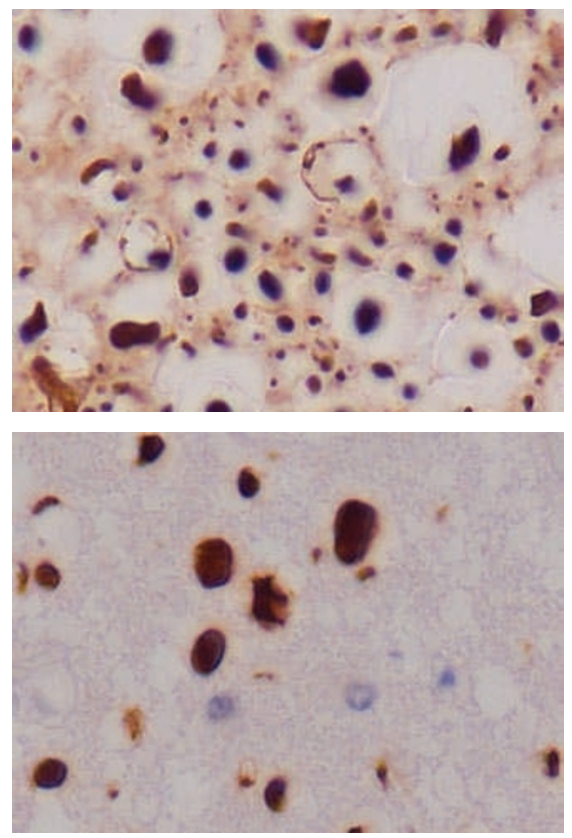

Immunohistochemical staining of corticospinal axons for NE14 in a patient able to mobilize with a stick (top) and a bedbound patient (bottom). Image provided by Dr Nikos Evangelou. 